\title{
Recanalização do ducto nasolacrimal com radiofrequência: estudo preliminar
}

\section{Recanalization of nasolacrimal duct with radio frequency: preliminary study}

\author{
Eduardo Alonso Garcia ${ }^{1,2}$, Marco Antonio Campos Machado², João Amaro Ferrari da Silva², Walton Nosé2
}

\section{RESUMO}

Objetivo: Avaliar a reconstituição do ducto nasolacrimal com o uso de radiofrequência para restabelecimento do fluxo lacrimal nos casos de obstrução da via lacrimal excretora.

Métodos: $O$ procedimento foi realizado em 16 olhos de 16 pacientes ( 13 femininos e 3 masculinos) pelo mesmo cirurgião, utilizando aparelho de radiofrequência monopolar de 450 kHz e 150 W de potência, com anestesia local sob sedação. Os critérios de inclusão foram obstrução baixa da via lacrimal (confirmada por dacriocistografia) e idade superior a 18 anos. Os critérios de exclusão foram trauma prévio, dacriocistite aguda, fístula cutânea, mucocele, cirurgia prévia da via lacrimal e uso de marca-passo cardíaco.

Resultados: O seguimento mínimo foi de 120 dias, os pacientes realizaram retornos ambulatoriais para avaliação clínica (presença de epífora, secreção, refluxo à expressão do saco lacrimal, posicionamento do tubo de silicone) e teste de Milder. Aos 90 dias de pós-operatório, 13 pacientes apresentavam irrigação positiva $(81,25 \%)$ com desobstrução do ducto nasolacrimal e 3 casos (18,75\%) de insucesso com irrigação impossibilitada (sem passagem para cavidade nasal). Conclusão: A reconstituição do ducto nasolacrimal com radiofrequência mostrou-se eficaz no tratamento da obstrução da via lacrimal excretora.

Descritores: Dacriocistorinostomia; Obstrução dos ductos lacrimais; Ducto nasolacrimal/cirurgia; Ondas de rádio

\begin{abstract}
Purpose: To evaluate the nasolacrimal duct reconstitution with radio frequency for restoration of lacrimal flow in cases of nasolacrimal duct obstruction.

Methods: The procedure was carried out in 16 eyes of 16 patients ( 13 women and 3 men) by the same surgeon, with monopolar high-frequency device at $450 \mathrm{kHz}$ and $150 \mathrm{~W}$, with local anesthesia under sedation. Inclusion criteria were lower lacrimal system obstruction (confirmed by dacryocystogram) and age over 18 years old. Exclusion criteria were previous trauma, acute dacryocystitis, cutaneous fistula, mucocele, previous lacrimal surgery and cardiac pacemaker.

Results: Patients were followed for at least 120 days, patients were clinically evaluated at outpatient clinics for the presence of secretion, epiphora, reflux at compression of the lacrimal sac, placement of silicone tube and Milder test. At the 90-day postoperative visit, 13 patients had positive irrigation (81.25\%) with clearance of lacrimal duct and 3 cases (18.75\%) presented irrigation failure.
\end{abstract}

Conclusion: Nasolacrimal duct reconstitution with radio frequency was effective in treating nasolacrimal duct obstruction.

Keywords: Dacryocystorhinostomy; Lacrimal duct obstruction; Nasolacrimal duct/ surgery; High frequency

\section{INTRODUÇÃO}

O tratamento da obstrução da via lacrimal excretora consiste em realizar uma nova passagem da lágrima para cavidade nasal por meio de uma osteotomia, comunicando o saco lacrimal à mucosa nasal. A via cirúrgica endonasal foi descrita antes da via externa no final do século XVIII (1). Algumas modificações foram realizadas nas técnicas cirúrgicas, e o avanço tecnológico introduziu novos instrumentos para realização destes procedimentos: fibroscópios, laser, brocas cirúrgicas, fibra óptica e o acesso transcanalicular pela miniaturização destes acessórios ${ }^{(1,2)}$.

Em 1994 a técnica de recanalização do ducto nasolacrimal (RCDNL) com radiofrequência (RF) foi publicada pela primeira $v e z^{(3)}$, descrevendo o restabelecimento do fluxo original da lágrima evitando a realização de osteotomia para recomunicar o sistema lacrimal com a cavidade nasal, interferindo minimamente na bomba lacrimal, sem causar cicatriz na face e lesão de estruturas do canto medial.

A RF é uma forma de corrente elétrica alternada cujo comprimento de onda varia de $3 \mathrm{kHz}$ a $300 \mathrm{GHz}$, e difere-se do restante do espectro eletromagnético pela capacidade de induzir movimento de partículas ionizadas. Uma antena transmissora emite as ondas que provoca uma corrente de elétrons em direção a uma antena receptora.

O efeito da energia eletromagnética no corpo humano depende da frequência utilizada: em baixa frequência a energia eletromagnética causa convulsão muscular (desfibrilador miocárdico); nas frequências mais elevadas a energia eletromagnética induz corrente que causa aquecimento nos tecidos que estão em contato com o eletrodo (bisturi elétrico). Em alta frequência o campo eletromagnético causa a polarização e oscilação das moléculas de água e a fricção entre essas moléculas transforma em calor. As configurações mais comuns na aplicação de RF na medicina são a monopolar e a bipolar.

Na RF monopolar a corrente elétrica é emitida por meio de um eletrodo aplicado à área de tratamento e retorna ao gerador por meio de um eletrodo de dimensões maiores localizado à distância (geralmente no dorso ou abdômen). A energia elétrica concentra-se próxima à ponteira do eletrodo ativo e diminui rapidamente com a distância.
Submetido para publicação: 1 de março de 2011

Aceito para publicação: 5 de fevereiro de 2012

Trabalho realizado na Santa Casa da Misericórdia de Santos - SCMS - Santos, SP, Brasill; e na UNIFESP - Universidade Federal de São Paulo - UNIFESP - São Paulo, SP, Brasil.

Médico, Setor de Plástica Ocular e Vias Lacrimais da Santa Casa de Misericórdia de Santos - SCMS Santos (SP), Brasil.

Médico, Departamento de Oftalmologia, Universidade Federal de São Paulo - UNIFESP - São Paulo (SP), Brasil.
Financiamento: Não houve financiamento para este trabalho.

Divulgação de potenciais conflitos de interesse: E.A.Garcia, Nenhum; M.A.C.Machado, Nenhum; J.A.F.Silva, Nenhum; W.Nose, Nenhum.

Endereço para correspondência: Eduardo Alonso Garcia. Rua Firmino Barbosa, 155 - Santos (SP) - 11055-130 - Brasil - E-mail: clios.oft@uol.com.br 
O objetivo deste trabalho é avaliar a eficácia desta técnica, isto é, a desobstrução do ducto nasolacrimal (comprovada pela irrigação da via lacrimal).

\section{MÉTODOS}

Foram realizadas 16 cirurgias de RCDNL com RF, associado à entubação com tubo de silicone médico (tubo lacrimal 0,9x0,6x 500 mm, Medikone ${ }^{\circledR}$, Brasil) para correção da obstrução da via lacrimal excretora, em 16 pacientes. Este estudo foi aceito pelo Comitê de Ética e Pesquisa (CEP) n 0463/10 da Universidade Federal de São Paulo (UNIFESP) - Escola Paulista de Medicina (EPM). Todos os pacientes leram e assinaram o termo de consentimento livre e esclarecido.

Os casos selecionados (13 femininos e 3 masculinos) eram provenientes do Departamento de Oftalmologia da Universidade Federal de São Paulo (UNIFESP) e da Santa Casa da Misericórdia de Santos (SCMS). Os critérios de inclusão foram: obstrução baixa da via lacrimal (confirmada por dacriocistografia) e idade superior a 18 anos.

Os critérios de exclusão foram trauma prévio, dacriocistite aguda, fístula cutânea, mucocele, cirurgia prévia da via lacrimal e uso de marca-passo cardíaco.

Os procedimentos foram realizados nas dependências da UNIFESP e da SCMS, pelo mesmo cirurgião, com aparelho de radiofrequência monopolar (WZC Lacrimal Recanalizer ${ }^{\circledR}$, Ghuangzhou, China) de $450 \mathrm{kHz}$, e $150 \mathrm{~W}$ de potência. A sonda do aparelho tem 1,2 mm de diâmetro x 140 mm de comprimento e sua extremidade (2 mm) é desprovida de isolamento.

Foi instituída sedação endovenosa e anestesia local (bloqueio anestésico) com xylocaína $2 \%$ associada a vasoconstrictor além de anestesia tópica, utilizando-se uma zaragatoa, embebida em xilocaína gel, sob o corneto nasal inferior.

Realizou-se a dilatação dos pontos lacrimais e a passagem da sonda de RF pelo ponto lacrimal superior por meio do ducto nasolacrimal até a cavidade nasal, tocando as hastes com pontas de algodão colocadas no meato nasal inferior (Figura 1). Aplicou-se $150 \mathrm{~W}$ de potência no ducto, movimentando a sonda ínfero-superiormente, até a transição com o saco lacrimal, evitando aplicar a RF dentro do saco lacrimal. Irrigou-se então o ducto nasolacrimal com água destilada (5 ml), confirmando a passagem livre para cavidade nasal sem resistência. Realizou-se a entubação pela técnica descrita em 2006 ${ }^{(4)}$ com catéter epidural 16 G (Portex Epidural Catheter, Smiths Medical, Reino Unido), bicanalicular e posteriormente a entubação com tubo de silicone médico utilizando o catéter como guia, suturado com fio de seda 6-0 (Ethicon ${ }^{\circledast}$, Brasil). Irrigou-se novamente com $5 \mathrm{ml}$ de solução de ciprofloxacino e dexametasona colírio diluído em água destilada no ducto nasolacrimal com Silastic ${ }^{\oplus}$ posicionado. As pontas do tubo de silicone foram cortadas no meato inferior e deixadas livres de sutura ou fixação.

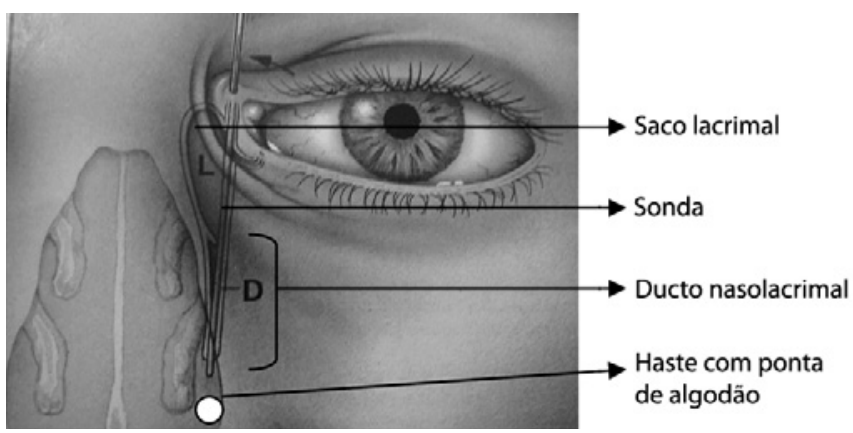

Figura 1. Fonte: Adaptada de Boyd BF. Atlas mundial de cirurgia oftalmológica. Cirurgia oculoplástica estética e reparadora. Edição brasileira. Highlights Ophthalmol. 1999;13(1):25. Figure 6-40.
Foram utilizados colírios de antibiótico em associação com corticoide (ciprofloxacino com dexametasona) por uma semana (4x/dia) e uso de solução salina na cavidade nasal associada a vasoconstritor por duas semanas. Foi prescrito também antibiótico oral (ciprofloxacino 500 mg de 12/12 h) por uma semana e anti-inflamatório (nimesulida $100 \mathrm{mg}$ ) oral por 3 dias no pós-operatório.

A irrigação do ducto nasolacrimal foi realizada inicialmente com 7 dias de pós-operatório, e posteriormente a cada duas semanas até o final do $2^{\circ}$ mês.

A evolução foi realizada nos retornos ambulatoriais pós-operatórios $(7,15,30,45,60,90$ e 120 dias) pelo quadro clínico e pelo teste de Milder, realizado com a instilação de colírio de fluoresceína $2 \%$ em fundo de saco conjuntival (em ambos os olhos) e análise subjetiva das características de escoamento do sistema lacrimal com lâmpada de fenda com luz de cobalto por 5 minutos. O menisco lacrimal desaparece muito lentamente ou permanece inalterado na presença de um bloqueio da drenagem. A intensidade da retenção é quantificada de zero a 4 cruzes, sendo zero e 1 cruz considerados normais ${ }^{(5)}$.

O tubo de silicone foi retirado após 60 dias da cirurgia, seguido de mais uma irrigação do ducto nasolacrimal. Considerou-se sucesso do procedimento quando a irrigação da via lacrimal era positiva (alcançava a cavidade nasal, sem resistência).

Foi solicitado controle radiográfico (dacriocistografia) após a retirada do tubo de silicone.

\section{RESULTADOS}

Todos os pacientes incluídos neste estudo apresentavam mais de 120 dias de pós-operatório.

Dos 16 pacientes, 13 apresentavam irrigação positiva, totalizando $81,25 \%$ de desobstrução do ducto nasolacrimal e 3 casos (18,75\%) de insucesso com irrigação impossibilitada no 90 dia pós-operatório (Tabela 1).

Uma paciente que não havia seguido a terapêutica prescrita apresentou dacriocistite aguda no 4 o dia de pós-operatório. Os sintomas regrediram com antibiótico oral. A mesma paciente retirou acidentalmente o tubo de silicone após 1 mês de cirurgia.

A dacriocistografia realizada após a retirada do tubo de silicone demonstrou passagem do contraste com discreto estreitamento na porção inicial do ducto nasolacrimal nos 13 pacientes com irrigação positiva (Figura 2).

\section{DISCUSSÃO}

Em 1994, publicou-se na China pela primeira vez o tratamento da obstrução do ducto nasolacrimal com a técnica de RCDNL com RF em 16 pacientes. $O$ estudo teve seguimento de 4 a 22 meses e apresentou taxa de sucesso de 93,8\% (irrigação positiva do ducto nasolacrimal)(3).

Desde essa época, a técnica de RCDNL com RF com frequência de $450 \mathrm{kHz}$ vem sendo utilizada por diversos grupos de pesquisadores chineses. Alguns estudos avaliaram o efeito das variações na potência, do uso de mitomicina C, dos locais diferentes de obstrução (canalículos, canal comum, obstruções associadas), porém a técnica utilizada sempre se resumiu em: desobstrução do ducto, por meio da retirada do material impactado e da mucosa adjacente, irrigação e entubação da via lacrimal com tubos de silicone e controle pósoperatório com irrigações semanais (3,4,6-10). $^{\text {. }}$

Tabela 1. Resultado funcional da recanalização do ducto nasolacrimal com radiofrequência no pós-operatório de 90 dias

\begin{tabular}{rlc}
\hline $\mathbf{N}$ & \multicolumn{1}{c}{ Resultado } & \% \\
\hline 13 & Irrigação positiva & 81,25 \\
3 & Irrigação impossibilitada & 18,75 \\
\hline
\end{tabular}



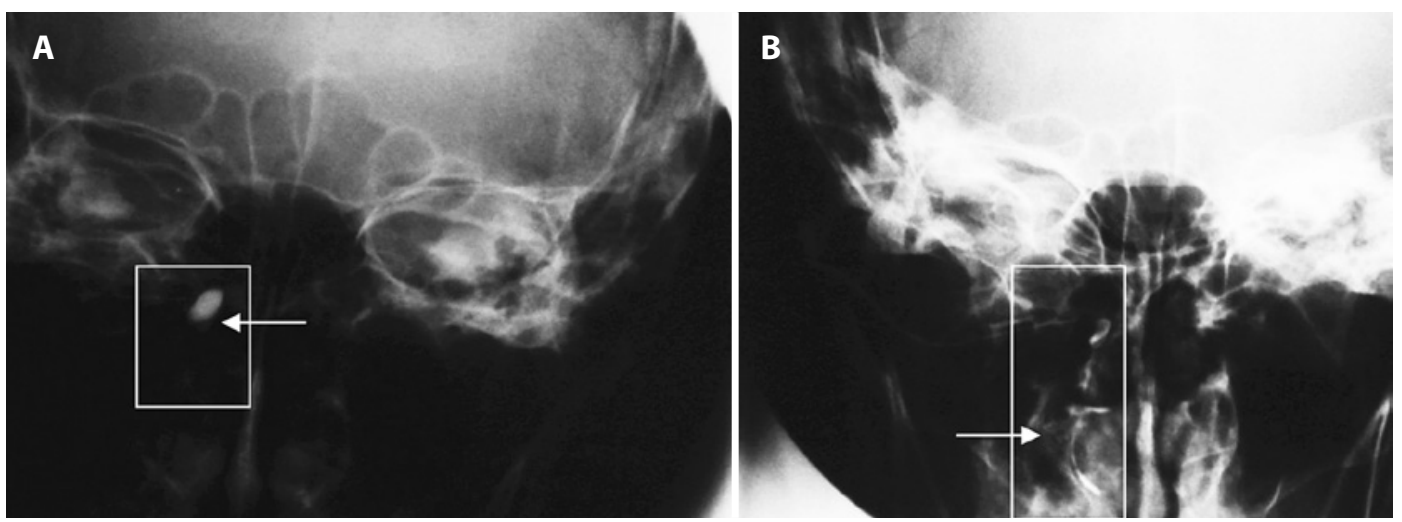

Figura 2. Dacriocistografias pré-operatória (A) e pós-operatória (B). Note a obstrução pré-operatória ao nível da válvula de Krause (seta) e a passagem do contraste pelo ducto nasolacrimal no pós-operatório (seta).

Em 1998 outros autores realizaram tratamento com RF em 79 olhos de 62 pacientes com obstrução da via lacrimal em diferentes sítios anatômicos com seguimento de 6 a 28 meses. As taxas de sucesso foram divididas em grupos; sendo 68,4\% (13/19) nos olhos com obstrução dos canalículos; $73,3 \%$ nos olhos com obstrução do canal comum (11/15); 86,1\% nos olhos com obstrução do ducto nasolacrimal (31/36), 40\% de sucesso em nos olhos obstruções altas e baixas (2/5 casos) e 100\% de sucesso nas obstruções de anastomose pós-dacriocistorrinostomia externa $(4 / 4)^{(6)}$

Em estudo que comparou os resultados do procedimento em 420 olhos (338 pacientes) utilizando potências de RF com magnitudes de $120 \mathrm{~W}$ e $140 \mathrm{~W}$, em diferentes grupo de obstrução (pontos lacrimais, obstrução alta, obstrução baixa, obstrução combinada, mais de 2 locais de obstrução) não foi detectada diferença estatisticamente significativa entre as potências utilizadas. A obstrução do ducto nasolacrimal ( $n=102$ ) apresentou taxas de sucesso de $90,2 \%$ e $98 \%$, com as diferentes potências, respectivamente ${ }^{(7)}$.

Esses estudos indicam a possibilidade de tratamento com a técnica de RF para obstruções altas, ou para os casos de obstrução baixa associados à obstrução dos canalículos.

Em estudo mais recente, pesquisadores apresentaram resultados do tratamento de obstrução baixa da via lacrimal com RF em 88 olhos (78 pacientes), com seguimento pós-operatório de 3 anos. Aos 3 meses de seguimento, a taxa de sucesso era de $72,1 \%$, diminuindo para $61,3 \%$ aos 12 meses $^{(8)}$.

A comparação da técnica de RF com e sem mitomicina C (MMC) foi realizada em estudo aleatorizado de 86 pacientes com obstrução do ducto nasolacrimal. Neste estudo o grupo controle (sem MMC) apresentou taxa de sucesso de $71,11 \%$, sendo estatisticamente inferior ao grupo com MMC, que apresentou $92,68 \%{ }^{(9)}$

O estudo com maior número de casos, publicado em 2009, incluindo 641 olhos de 583 pacientes, com seguimento médio de 28,5 meses (variação de 12 a 54 meses) comparou a RCDNL por RF (506 olhos, 457 pacientes) com a dacriocistorrinostomia (DCR) por via externa (135 olhos, 126 pacientes). Não foram detectadas diferenças entre os resultados das duas técnicas. Foi considerado sucesso total quando o paciente não apresentava sintomas (86,96\% para RCDNL e $87,41 \%$ para DCR), sucesso parcial quando existia epífora, mas com irrigação positiva (6,13\% para RCDNL e 3,70\% para DCR), e falência quando além da epífora, ocorria secreção, refluxo à expressão do saco lacrimal ou irrigação impossibilitada após a retirada do tubo de silicone (6,92\% para RCDNL e 8,89\% para DCR) $)^{(10)}$. Este estudo também avaliou a histopatologia em macacos após a aplicação da RF. Os resultados demonstraram a recuperação total do epitélio do ducto nasolacrimal em 1 mês e após 2 a 3 meses a ausência de células inflamatórias no tecido.
Em nosso estudo realizamos o procedimento e o controle pósoperatório como descrito nos trabalhos $\operatorname{citados}^{(10)}$. O seguimento do trabalho, com uma amostragem maior e um acompanhamento mais longo, deve oferecer mais dados para análise. Numa segunda fase, realizaremos a RCDNL com RF e tubo de silicone, porém sem as irrigações no controle pós-operatório para analisar se este procedimento influi no resultado da cirurgia. Uma avaliação sobre as doenças nasais pré-existentes (rinite, sinusite) e as alterações do filme lacrimal (hipo e hipersecreção) pode também auxiliar no estudo da eficácia imediata e tardia da cirurgia. Após a análise das obstruções baixas, as obstruções altas e mistas da via lacrimal serão o objetivo dos trabalhos subsequentes.

\section{CONCLUSÃO}

A recanalização do ducto nasolacrimal com radiofrequência mostrou-se eficaz em 81,25\% dos casos (pacientes com irrigação da via lacrimal positiva pós-operatória).

\section{AGRADECIMENTO}

Ao Prof. Dr. Zhichong Wang, em nome da equipe do Ocular Surface Center, State Key Laboratory of Ophthalmology (Zhongshan Ophthalmic Center, Sun Yat-sen University - Guangzhou - China) pela instrução da técnica de recanalização do ducto nasolacrimal com radiofrequência.

\section{REFERÊNCIAS}

1. Cintra PP. Dacriocistorrinostomia endocanalicular com laser de diodo [tese]. Ribeirão Preto: Faculdade de Medicina de Ribeirão Preto da Universidade de São Paulo; 2006.

2. Garcia EA. Complicações na dacriocistorrinostomia transcanalicular com laser diodo. Arq Bras Oftalmol. 2009;72(4):493-6

3. Wang ZC, Chen D, Cheng PZ. [Endolacrimal high-frequency electric cauter in treatment of chronic dacryocystisis]. Chin J Ophthalmol. 1994;30:230-1. Chinese.

4. Wang L, Chen D, Wang Z. New technique for lacrimal system intubation. Am J Ophthalmol. 2006;142(2):252-8.

5. Zappia RJ, Milder B. Lacrimal drainage function. 2. The fluorescein dye disappearance test. Am J Ophthalmol. 1972;74(1):160-2

6. Yao DQ, Li L, Liu CM. A therapy of endolacrimal high-frequency electric cautery for dacrogatresia. Ophthalmol China. 1998;7:95-7.

7. Chengzhi X, Yongming Y. Treatment of lacrimal passage obstruction by high frequency electric cauterization combined with perfusion of antibiotic gel. Chin J Pract Ophthalmol. 2006;24(5):545-8.

8. Sun X, Shan B. Treatment of chronic dacyocystitis by high frequency electric cautery J Preclin Med Coll Shangdong Univ. 2002;16(5):303-4.

9. Nan YE, Hou GH, Xu JT, Xu H, Xue Y, Liu XH. [High frequent electrolacryocystoplasty combined with mitomycin on obstruction of naso-lacrimal duct]. Recent Adv Ophthalmol. 2008;(12):913-4. Chinese.

10. Chen D, Ge J, Wang L, Gao Q, Ma P, Li N, et al. A simple and evolutional approach proven to recanalise the nasolacrimal duct obstruction. Br J Ophthalmol. 2009;93(11): 1438-43. Comment in: Br J Ophthalmol. 2009;93(11):1416-9. 